\title{
DeePay: Deep Learning Decodes EEG to Predict Consumer's Willingness to Pay for Neuromarketing
}

This paper was downloaded from TechRxiv (https://www.techrxiv.org).

\section{LICENSE}

CC BY 4.0

SUBMISSION DATE / POSTED DATE

24-03-2021 / 09-04-2021

\section{CITATION}

Hakim, Adam; Golan, Itamar; Yefet, Sharon; Levy, Dino J. (2021): DeePay: Deep Learning Decodes EEG to Predict Consumer's Willingness to Pay for Neuromarketing. TechRxiv. Preprint.

https://doi.org/10.36227/techrxiv.14287427.v2

$\mathrm{DOI}$

10.36227/techrxiv.14287427.v2 


\title{
DeePay: Deep Learning Decodes EEG to
}

\section{Predict Consumer's Willingness to Pay for}

\section{Neuromarketing}

\author{
Adam Hakim¹, Itamar Golan², Sharon Yefet ${ }^{3}$, Dino J Levy ${ }^{1,3^{*}}$ \\ ${ }^{1}$ Sagol School of Neuroscience, Tel Aviv University; ${ }^{2}$ Blavatnik School of Computer Science; ${ }^{3} \mathrm{Coller}$ \\ School of Management, Tel Aviv University \\ ${ }^{\star}$ corresponding author: dinolevy@tauex.tau.ac.il
}

\begin{abstract}
There is an increasing demand within consumer-neuroscience (or neuromarketing) for objective neural measures to quantify consumers ' preferences and predict responses to marketing campaigns. However, the properties of EEG raise difficulties for preference prediction: small datasets, high dimensionality, elaborate manual feature extraction, intrinsic noise, and between-subject variations. We aimed to overcome these limitations by combining unique techniques within a Deep Learning (DL) framework, while providing interpretable results for neuroscientific and decision-making insight. In this study, we developed a DL model to predict subject-specific preferences based on their EEG data. In each trial, 213 subjects observed a product's image, from 72 possible products, and then reported how much they were willing to pay (WTP) for the product. The DL employed EEG recordings from product observation to predict the corresponding reported WTP values. Our results showed $75.09 \%$ accuracy in predicting high vs. low WTP, surpassing other models and a manual feature extraction approach. Meanwhile, network visualizations provided the predictive frequencies of neural activity, their scalp distributions, and critical timepoints, shedding light on the neural mechanisms involved with evaluation. In conclusion, we show that DLNs may be the superior method to perform EEG-based predictions, to the benefit of decision-making researchers and marketing practitioners alike.
\end{abstract}

Index Terms- Computational neuroscience, Neural nets, Marketing, Physiological signal

\section{Introduction}

In the last decade, the field of consumer neuroscience, or neuromarketing, is flourishing, with numerous publications, academic programs, initiatives, and companies. There is a growing community of scientists conducting studies in the field that converged into multiple meta-analyses and reviews [1], [2], [11]-[15], [3]-[10]. There is an increased demand for objective neural measures that quantify consumers' preferences and predict responses to marketing campaigns. Many researchers and practitioners aspire to measure neural and physiological activity to predict a future decision or action of an individual or to assess the success of possible marketing campaigns in the general population. This aspiration is driven by the limitations of traditional marketing techniques, such as questionnaires, focus groups, and interviews [16].

One candidate for acquiring neural information is Electroencephalography (EEG) - an electrophysiological method that records the electrical activity of the brain by attaching several electrodes (electrical conductors) along the scalp [17]. EEG recordings provide an approximation of neurotransmitter-mediated neural activity, with high temporal resolution (milliseconds) but poor spatial resolution [18]. This technique is commonly used in the neuromarketing industry (see NMSBA Website), and there is accumulating evidence linking various EEG signals with value-based choice [19]-[23]. Several academic studies have already employed EEG recordings to predict subjects' stated preferences or actual choices [24], [25], [34], [35], [26]-[33], or population marketing success [36]-[43]. However, only a few tried to use the latest computational modeling techniques. Importantly, previous studies relied on manual feature extraction and had small data sets.

For example, a recent study recorded EEG data from 30 participants as they watched 220 different advertising commercials, and used an SVM model on manually extracted features to predict participants' questionnaire responses that capture their purchase intent [30]. Another study predicted the effectiveness of SuperBowl commercials based on features extracted manually from the EEG signals alongside eye tracking and heart rate data [40]. The authors used a simple neural network with only two fully-connected layers with ten and five units, respectively, and attempted to predict the number of YouTube viewings for each commercial. Additionally, a team from Neuromarketing Labs reported they could predict, using features manually extracted from the EEG signals, between successful and unsuccessful sales of shoes. They used a custom-made "preference index computed through an internally developed algorithm loosely associated with parameters from basic emotional neuroscience" [44]. Another study applied a hidden Markov model on features they extracted from the EEG signals of 40 participants while they were watching images of 14 different products, to predict their likes/dislikes of the products [25]. The authors also tested various other models, such as support vector machine, random forest, and nearest neighbors.

A different study applied a probabilistic neural network and k-nearest neighbors prediction models [45] to predict preferences. In that study, participants viewed four commercials per four different vehicle brands while their EEG activity was measured and processed manually to several features. Lastly, a study conducted in our lab used a combination of a multitude of EEG measures for preference 
prediction that we manually extracted based on previous literature: inter-subject correlations (ISC), hemispheric asymmetry, and spectral power bands [46]. Subjects watched six different product commercials while we recorded their EEG activity. We predicted both subjects' choices and the commercials' success at the population level. Importantly, we showed that adding the EEG data increased the prediction success compared to what we could achieve when using only a standard marketing questionnaire. To accomplish this, we utilized various machine learning models, such as support vector machines, decision trees, kernel discriminant analysis, and more.

Although these studies demonstrated successful predictions, they all manually extracted the features of the EEG signal. Moreover, each study used a different combination of features as the input to their model. Hence, as a scientific community, this prevents us from converging to well-established and generalizable EEG-based neural signals that represent value across various domains, stimuli, and environments. To overcome these shortcomings, in the current study, we use a state-of-the-art deep learning model both as our predictive algorithm and as our feature extraction procedure.

The clear dogma that emerges from examining the previous EEG-based preference prediction studies is that they manually extracted various features from the EEG signal and used some classifier to learn the conditional probability of preferences on the extracted features. As shown above, the features extracted rely on previous findings relating these features to different aspects of the valuation process [16]. We propose that this approach to preference prediction is fundamentally limited and suffers from issues constraining its success.

First, the properties of EEG datasets, in general, raise difficulties for conventional machine learning and regression methods in prediction. This is because the size of the EEG datasets is often small, the EEG signal is high dimensional, very noisy, obscure, non-stationary, and with high unpredictable variations between subjects. Moreover, there is a need for elaborate and exploratory feature extraction.

Second, in all previous attempts, the experimenters chose in advance, based on previous studies, which EEG features to use for preference prediction. Thus, researchers limit the information that could be extracted from the EEG recordings to whatever can be captured based on their specific predefined pre-processing and feature extraction pipelines. However, there are unlimited possibilities of features to use and pre-processing parameters to tune manually. For example, assume we want to use theta frequency power band (which is in the range of $4-8 \mathrm{~Hz}$ ) as an input to the model. One question that immediately arises is whether to take the entire range from $4-8 \mathrm{~Hz}$, or maybe just the range between $5-7 \mathrm{~Hz}$, or perhaps allow each subject her unique range. Moreover, researchers must choose various parameters with which to conduct the spectral transformation, such as the time window, overlap, frequency resolution, and transformation technique (wavelet, STFT, etc.). These questions only scratch the surface, as there are endless possibilities and free parameters to decide from what time points to extract any feature. On top of that, we can add the variety of options available for treating specific electrodes and the signal's spatial components (Averaging, maximizing, ICA, etc.).

There are numerous possibilities in each feature extraction pathway, giving the researcher countless degrees of freedom. It is almost impossible to know in advance which exact feature, pre-processing method, and extraction procedure will give the best prediction. This allows the researcher to explore various procedures and arrive at the most predictive features for his/her dataset, which could easily result in overfitting the dataset and failing to generalize the results on new data. We propose that this might explain the limited success of EEGbased features in out-of-sample predictions. In fMRI, the degrees of freedom during the pre-processing stage were estimated to be in the order of tens of thousands [47], and we contend that EEG is not different in this matter.

Lastly, and this is mainly a problem for regression approaches, even if for some miraculous reason we can choose the best possible features without overfitting, we still need to figure out if there are interactions between these features and what exactly they are. For example, it is often unclear whether we need double or triple interactions between each feature in the model, or whether their interaction may be non-linear, and so on.

To overcome these limitations, in this study, we use a Deep Learning (DL) network [48] that combines several state-of-theart techniques. Thanks to their superior ability to learn complex representations and automatically extract optimal features, DL networks have revolutionized many research fields, such as Computer Vision, Brain-Computer Interfaces, Artificial Intelligence, and more. Importantly, DL networks have already shown success utilizing EEG signals for many applications [49], [50].

The applications vary from epilepsy prediction and seizure monitoring [51]-[58], to auditory music retrieval [59], [60], detection of visual-evoked responses [61]-[64], mental workload classification [65], [66], sleep stage recognition [67][71], emotion detection [72]-[79], limb control [80], biometric authentication [81], and motor imagery classification [82][91]. Moreover, a review of 154 papers found that the median gain in accuracy of DL approaches compared to regression and other machine learning models was 5.4\% [92].

In our study, we developed a novel DL network architecture that analyses EEG neural signals in order to predict individual preferences. Since DL networks require a large amount of data, we collected an extensive dataset that has over $35 \mathrm{k}$ samples of EEG recordings from 213 subjects. We acquired the EEG recordings while subjects observed pictures of products and then reported their subjective valuation of the observed product. Then, we applied a DL model to overcome the drawbacks of EEG measurements stated above and optimally performed predictions of subject-specific value preferences. We combined several state-of-the-art techniques that alleviate the need to manually search for features in the EEG signal that contain value-related information, without a priori choosing any features or preprocessing pipelines. Lastly, we used network visualization techniques to gain insights regarding the attributes of the signal that best predict value and thus the neural mechanisms involved.

\section{Methods}

\subsection{Subjects}

To our knowledge, none of the BCI, EEG, or Neuromarketing communities have published a dataset with EEG recordings and corresponding valuations of products in the scale appropriate for deep learning. Therefore, we have undertaken the task of creating such a dataset in our own laboratory. Two 
hundred and thirteen subjects (96 males) participated in the study, aged 19-51 (Mean =25.36, STD =5.07). We excluded 30 subjects from the analysis based on our strict exclusion criteria (elaborated later) and a single recording failure, resulting in a total of 183 subjects. All subjects gave written informed consent before participating in the study, which was approved by the local ethics committee at our university.

\subsection{Stimuli}

Stimuli consisted of 72 pictures of products from 6 different categories (12 products per category). In addition to the picture of the product, there was a short text above the picture describing the product. See Appendix A for a full list of products. Products were chosen such that their real-world cost exceeded the maximal bid amount, incentivizing subjects to bid for the products regardless of any cost-benefit calculation related to their real-world value, but rather to reflect their subjective value. Their market price ranged from 45 to $99 \mathrm{New}$ Israeli Shekel (NIS, Mean $=69.23$, STD $=16.05$ ).

\subsection{Experimental Design}

For each subject, we first presented the full experimental instructions and applied a short verbal test to verify they understood the task. They received 50 NIS as a participation fee and an additional 50 NIS as the endowment for the behavioral task. Then, we mounted them with an 8-electrode (wet) EEG system (StartStim 8 system by Neuroelectrics, Spain) at positions F7, Fp1, Fpz, Fp2, F8, Fz, Cz, Pz, sampled at $500 \mathrm{~Hz}$. Subjects were instructed to sit upright in front of the computer screen, maintain their position with minimal movements throughout the task. As can be seen in Fig. 1, on each trial, while subjects watched each product on the computer screen, they were instructed to think how much they value the product. Each product was presented for 3.5 seconds. Afterward, a horizontal sliding bar appeared, and subjects stated their maximal amount of money they were willing to pay for the product (WTP), between 0 and 50 NIS ( 14.5\$). Subjects had up to 15 seconds to state their WTP. The sliding bar was accompanied by the instruction, "Please indicate how much you would be willing to pay for the product". When a subject understands the task correctly and the BDM procedure, their WTP bid should correspond to her subjective value for the product.

Importantly, we randomly initialized the location of the "tick" that indicates the chosen amount on the sliding bar, such that subjects did not know in advance whether and how far they would be moving the cursor left or right on the sliding bar after seeing the product. We did this to avoid motor preparation signals in the EEG recordings, which could be correlated with value and obscure our desired value signal. Lastly, a black screen appeared as an inter-trial-interval (ITI) for one to two seconds (duration chosen at random uniformly), and the subsequent trial would commence. See Fig. 1 for an illustration of the experimental procedure. Every 20 trials, a screen appeared with the instruction "Remember that you must think of the value of the product as it appears before you", which required a mouse click to resume. This screen served as a reminder for subjects to think of the value of the products as they observe them, in hopes that it strengthened the value signal in the EEG recordings. Subjects viewed each product 3 times for a total of 216 trials in random order.

We applied the standard Becker-DeGroot-Marschack $(\mathrm{BDM})$ procedure [93] in order to make the experiment incentive compatible. That is, after the experiment was finished, one trial was chosen at random. The computer randomly generated a price for the product of the chosen trial, between 0 to 50 NIS. If the amount the subject offered for the product in that trial was larger than the random price - then the subject received the product at the random price and kept the remaining change from his endowment. A subject's total winnings, in this case, were the product, the 50 NIS participation fee, and the difference between the 50 NIS endowment and the random price. If the amount the subject offered was lower than the random price generated, then the subject did not win the product and kept the endowment in its entirety, making his total earnings 100 NIS.

Fig. 1. Experimental Design. Subjects first received instructions and were mounted with an 8-electrode EEG cap. Then, they watched 72 products, three times each. After each product viewing, they stated how much they were willing to pay for the observed product, from 0 to 50 NIS.

\subsection{EEG Preprocessing}

The EEG recordings were down-sampled by 2 (from 500 hertz to 250 hertz) and divided to separate epochs, such that each recording during a product observation served as a single sample in the dataset. An epoch included 3.5 seconds of product observation and 1 second from the previous ITI, which the model used as the baseline. We discarded the recordings while subjects stated their WTP values, so we would not confound a signal for subjective value with motor execution. The EEG sensors could pick up the cortical activity related to moving the mouse cursor to a selected WTP value, so removing this motor information from the data assured that our model would only identify value-related information. Thus, a sample consisted of 8 channels and 1125 timepoints ( 4.5 seconds sampled at 250 hertz).

We wanted to minimize any manual pre-processing and filtering, so we could provide the model with the closest data to a raw signal and have the model perform any preprocessing within it. Hence, the only pre-processing steps we conducted was first, to apply a bandpass filter on the whole experiments' recording of each electrode separately (before dividing into epochs), between 0.5 hertz and 100 hertz (second-order Butterworth), and second, we subtracted the mean signal per sample per electrode. Moreover, we defined criteria to exclude samples with excessive noise. For a given sample, any electrode recording with a standard deviation higher than $50 \mathrm{mV}$ or with a maximal amplitude higher than $400 \mathrm{mV}$ was discarded and replaced by a neighboring electrode [94]. If a given sample had four or more electrodes, which correspond with these exclusion criteria, then the entire sample was discarded. Out of all samples from the relevant subjects, $7.37 \%$ of the samples were removed following these criteria. One subject was removed due to a failure in the recording.

Aside from preparing the EEG data for the network, we also wanted to inspect the data outside of the network, so we created spectrograms of each sample. We transformed each trial's EEG signal recorded from the FPZ electrode to a spectrogram, using MATLAB's "spectrogram" function. We used a window size of 1.5 seconds, with 0.01 seconds overlap between windows, and inspected frequencies between $0.5 \mathrm{~Hz}$ to $70 \mathrm{~Hz}$ (with a $0.5 \mathrm{~Hz}$ interval). The result was the Power Spectral Density $[\mathrm{W} / \mathrm{Hz}]$ for every time window and 
frequency, also termed a "spectrogram", for each trial.

\subsection{Behavioral Preprocessing}

We enforced several behavioral exclusion criteria, according to subjects' WTP values, in order to ensure that the task was able to elicit genuine subjective preferences in our subject pool. Including subjects that did not perform the task seriously or sincerely would damage our dataset and hinder our model's ability to identify the relationship between the neural signal and actual individual value. If a given subject provided WTP values smaller than 5 NIS $(10 \%$ of the maximal amount) for more than half of her trials, the subject was excluded entirely from the dataset (Fig. 2A). This type of subject had demonstrated that she was disinterested in most of the products offered in the task and therefore could not provide a reliable value signal in her recordings. Additionally, we also tested whether a given subject exceeded 45 NIS $(90 \%$ of the maximal amount) for more than half of her trials (Fig. 2B). This subject would likely be over-enthusiastic to purchase any item without weighing their subjective value properly.

Additionally, we tested the correlation between the WTP values for the same items in the second and third blocks, which we expected to be the highest between-block correlation for a given subject, as they have most likely settled on the value of each product. We excluded subjects with a correlation coefficient lower than 0.5 (Fig. 2C) since they seemed far apart in their coefficient compared with the rest of the subjects, which at least arrived at 0.74 (Fig. 4). These subjects were too inconsistent in their valuations, raising suspicion that their reported valuations were closer to random. Twenty-nine subjects failed in at least one of these criteria, bringing our subject pool to a total of 183 subjects. For all these excluded subjects, the task failed to elicit varied subjective valuations for the products presented, so their exclusion does not reflect any shortcomings of the DL network and its predictions. Finally, we divided all WTP values by 50 (the maximal amount) to normalize them to the range between 0 to 1 . Each WTP value served as the label for the EEG recordings of its corresponding product observation. Thus, we attempted to learn and predict these WTP values based on the EEG recordings as specified in the previous paragraph.

Fig. 2. Behavioral Exclusion Criteria. These graphs demonstrate the WTP values for all three blocks, sorted by the second block (lowest to highest value), for four example subjects. (A) Subject 12 was excluded since she bid less than 5 NIS over $85 \%$ of her trials, representing very low variability in WTP across products. We excluded 27 subjects based on this criterion. (B) Subject 40 was not excluded and is considered a good subject. Note that she bid above 45 NIS in only $47 \%$ of her trials and was therefore not excluded. No subjects were excluded based on this criterion. (C) For subject 149, the correlation between the second block and third block was 0.41 , representing very high variability in WTP values. This might suggest that this subject did not report its "true" valuations but answered randomly or that her actual valuations change very quickly on a block-byblock basis. Two subjects were excluded based on this criterion. (D) Subject 172 exhibited consistent behavior across the three repetitions of the BDM blocks and, in addition, highly varied valuation across products. Her correlation coefficient between blocks 2 and 3 was 0.97 , her bid rate below 5 NIS was only $12 \%$, and her bid rate above 45 NIS was only $8 \%$.

\subsection{Network Architecture}

We propose a proprietary DL network architecture that considers the unique characteristics of the EEG signal and offers several types of processes and components that account for the signals' spectral, spatial, temporal, and subject-specific dependencies. This unique architecture accepts the raw EEG signal as input, implements classic signal processing procedures to extract features automatically, provides interpretability throughout these steps, and finally outputs a prediction of preference. In the first layer, temporal filters are applied to the raw EEG multi-channel signal. These create separate "spectral maps" per each filter. Then, a spatial convolution is performed, accounting for the information from different channels. Afterward, spatial maps are summed, resulting in time-series feature maps. These are fed into recurrent layers and an attention mechanism that captures the relationships between the different temporal sections of the representations extracted from the previous layers. Finally, we apply a SoftMax activation function on the final two nodes of the network for the probability of that sample to belong to each class (=High/Low WTP), or we directly output a final node as a single regression output. As meta procedures, we optimized our hypermeters using the Parzen Estimator approach (TPE) [95] and used an ensemble of networks with random initializations for each prediction.

\section{Results}

\subsection{Behavioral Results}

Before fitting a neural network to the data, we wanted to examine the general behavior in the BDM task and whether valuations of each product varied between subjects, and if subjects were consistent with their valuation of a product across blocks. It is problematic if all subjects have similar WTP values across all products since then predicting a product's value could be a matter of identifying its image's particular pattern of neural response, rather than basing the prediction on the subjective value signal it elicited for each subject. If this was the case, the model could hypothetically use the similar WTP values across subjects at the product's identifier and employ shared neural responses to the product for its prediction instead of predicting each subject's unique valuation. We addressed this possible problem, in part, by including 72 products in the experiment. This makes it very difficult for a model to hypothetically learn 72 unique product identifiers based on common neural responses to products but instead makes it more likely for the model to learn neural value representation.

However, to negate this possible problem even further, we inspected how much the WTP values varied across subjects. Therefore, per product, we averaged all valuations from all subjects and blocks. As can be seen in Fig. 3, there was a nice gradual increase in WTP across products averaged over subjects. Products from the "Experience" category were the most preferred products, as six products from this category had the highest WTPs. In the bottom end, the "Office" category was most prevalent, with seven products from that category at the bottom 15 least preferred products. Notably, the WTP values spread around the average of each product, such that no value could be said to correspond to a singular product uniquely. To further substantiate this claim, we tried to predict High/Low WTP values (using the same procedure as in the subsection "WTP Prediction"), based on the item name and its category, coded as dummy variables (or one-hot vectors). If product identity did not predict WTP values, then it would be highly improbable that EEG-based prediction of WTP was mediated by product identity. We reached $58 \%$ 
prediction accuracy using logistic regression, showing that the identity of products could predict WTP values to a small degree, but not nearly enough to explain the EEG-based predictions of WTP that we demonstrated with our model in the following subsections.

Regardless of this issue, we could also see that subjects seemed to prefer bidding rounded values, with zero and 50 being most prominent, but also 5, 10, 15, and so on. This can be seen on the histogram chart that appears on the right of Fig. 3, which shows the relative frequency of each WTP value across all products and subjects.

Fig. 3: WTP Values per Product. The above shows the average WTP values across subjects and blocks as "filled" large dots, and the actual values scattered around them as semi-transparent smaller dots, so a more popular value for a product will appear as more saturated and denser dots. The right of the graph shows the frequency of each WTP value across all products and subjects, binned to a width of 1 NIS. The percentages signify the propensity of all WTP values binned to a width of 10 NIS each, from 0 to 50. Error bars signify the standard error of the mean (SEM).

Next, we wanted to examine the consistency of valuations between the three different blocks to estimate whether subjects reported persistent values for each item they saw. This analysis was used in the mentioned exclusion criteria, and enabled us to examine whether subjects were engaged and sincere in the task, as would be reflected by consistent valuations between blocks. For each subject, we calculated three Pearson correlation coefficients across all the valuations of products; Between their valuations in the first and second blocks (Mean $=0.845$, STD $=0.087)$, between the first and third blocks (Mean $=0.821$, STD $=0.096)$, and between the second and third blocks (Mean $=0.911, \mathrm{STD}=0.072$ ). We found that $99 \%$ of all correlations for all subjects were above 0.5 , while $90 \%$ of correlations were above 0.75 , and $38 \%$ of all correlations were above 0.9 . This demonstrates that subjects were highly consistent in their valuations across blocks. In the exclusion criterion for this analysis, we used only the correlation between the second and third blocks to exclude subjects. This was because we estimated that these correlations should be the highest across our sample since subjects should converge to a constant valuation of a product the more times they evaluate it. Therefore, a subject with an exceptionally low correlation between her second and third blocks was more likely to be a candidate for exclusion. To corroborate our approach, we performed one-way dependent t-tests, hypothesizing that subjects' correlation between the second and third blocks are higher than the other correlations. Indeed, correlations between block 2 and 3 were significantly larger than correlations between blocks 1 and $2(t(181)=8.39$, $\mathrm{p}<0.001)$ and blocks 1 and $3(\mathrm{t}(181)=10.15, \mathrm{p}<0.001)$, as can be seen in Fig. 4.

Fig. 4: Correlation of Valuations Between Blocks. The correlation between valuations of the same products between every two blocks, per subject. Correlations between blocks 2 and 3 were significantly larger than correlations between blocks 1 and $2(t(181)=8.39, p<0.001)$ and blocks 1 and $3(t(181)=10.15, \mathrm{p}<0.001)$.

\subsection{Network Results}

\subsubsection{Experiment Parameters}

We performed the hyperparameter tuning procedure to set various parameters of our network. The TPE process was evaluated with a validation dataset consisting of $10 \%$ of the data and provided the hyperparameters we used for the final predictions on the test set. These parameters include kernel sizes, number of filters, dropout probability, learning rate, batch size, and more. The dropout probability was set to 0.23 throughout the network, the learning rate was set to 0.001 , and we used the standard "Adam" optimizer.

\subsubsection{WTP Prediction}

We conducted several predictions to address our main aims. We wanted to test the ability of the network to conduct both binary classification and continuous predictions (by simply removing the final SoftMax layer in the network). First, we divided the dataset into $80 \%$ training, $10 \%$ validation, and $10 \%$ testing. The validation set was used for the hyperparameter tuning (TPE) procedure and for early stopping during training. For training the network in all binary prediction attempts, we took the bottom $0-35 \%$ of WTP values and labeled them as class 0 , while the top $65-100 \%$ was labeled as 1. For training the network for continuous value predictions, we used the same data but with the original WTP values. The rest of the samples in the training set were discarded (35-65\% WTP). This was done to boost the network's ability to identify distinct value information while learning, since the $35-65 \%$ of WTP values are the closest in value terms, and hence, would be the hardest between which to distinguish. This was also reflected in another analysis we did, where the two midranged quartiles were much more similar to one another than the two quartiles further apart (See Fig. 8 \& Fig. 9 below).

For all binary predictions, we report the area under the curve (AUC) score and the accuracy score, while for the continuous predictions, we report the root mean square error (RMSE) score. We report prediction scores that are based on the $10 \%$ held-out test set that was randomly pooled out from the entire dataset before training. We conducted several binary predictions, wherein each prediction attempt, the test set was divided based on different WTP percentiles. That is, we report accuracy, AUC, and RMSE on prediction of the top and bottom $20 \%$ WTP values $(0-20 \% / 80-100 \%)$, then on the following $15 \%$ (20-35\% / 65-80\%), then the middle $15 \%$ (35$50 \% / 50-65 \%$ ), and finally the overall scores for the entire range of WTP values within the test set. We segmented the reported results in this fashion to provide a better understanding of the model's performance across diverse levels of difficulties in prediction, instead of only providing result metrics over all the data. We would expect that prediction between the bottom and top 20\% WTP values would be easiest and therefore more accurate, as the neural representation would be more distinct. This is in line with the notion of "neural distance", as detailed in our previous study [24], [46], [96]. The most adjacent $15 \%$ of WTP values would be, therefore, harder to predict. For clarification, all reported prediction scores were based on the same single model that was trained on the bottom $0-35 \%$ and the top $65-100 \%$ WTP values out of the training set, for either binary or continuous predictions.

In order to test the general success of the prediction results of our network, we compared them to the prediction results of several state-of-the-art benchmark techniques and other more standard approaches. First, we shuffled the labels with respect to the samples to obtain a baseline "random" prediction. Next, we manually extracted the same features from the EEG data 
and used the same procedure as in our previous study [46]. Namely, we extracted the inter-subject correlation (ISC), the spectral power, and the hemispheric asymmetry for all five standard frequency bands (Delta, Theta, Alpha, Beta, Gamma). Additionally, we extracted the three ERP components we identified in the current study (see results of our ERP analysis below) to increase the prediction capabilities of these manually extracted features. We used XGBoost [97], SVM, and Linear/Logistic Regression (depending on whether it was a binary or a continuous value prediction) as the machine learning models. Out of the three models we tested, XGBoost scored the highest, and therefore we present it here and compare it to the performance of our network. We used the model with the manual features extracted alongside the machine learning model as a legitimate contestant to the automatic feature extraction performed via our deep learning network. We think that this comparison can test if our claim regarding the superiority of the DL framework in prediction holds. We also added two well-known and often used deep learning models - DeepCovNet [82] and EEGNet [98]. The first is a standard convolutional neural network, and the second is a popular network with architecture specialized for EEG decoding and prediction. We also optimized all the additional models using the same TPE procedure we used for our network.

Results in Table 1 show that our DL network, termed DeePay, achieved better prediction scores compared to the other DL networks, the classic machine learning model using manual feature extraction, and the model of shuffled labels. Only when trying to differentiate between the percentiles closest to each other and to the median (i.e., a binary classification between $35-50 \%$ and $50-65 \%$ ), our DL framework did not outperform other models, as differentiating between such close preferences was a difficult challenge for any model. Indeed, no model achieved significant results for this slight percentile difference, and their prediction accuracies were most likely obtained by chance, yielding no superior model.

Table 1: Prediction Results. Accuracy, Area under the curve (AUC), and Root Mean Square Error (RMSE) for seven different prediction procedures/models, as detailed in the left-most column. We report each of these scores for four different subsets of the dataset - the first and last 20\% of WTP values in the data $(0-20 \% / 80-100 \%)$, the next $15 \%$ of WTP values from the top and bottom of data (20-35\% / 65-80\%), the following $15 \%$ that represent the center of WTP values (35-50\% / 50-65\%), and finally the results on the entire dataset.

\subsubsection{Network Interpretability}

Deep learning models are typically regarded as black boxes. Although these models reach impressive prediction accuracies, their nested non-linear structure makes them highly non-transparent. That is, it is not clear what information in the input data makes the model arrive at its predictions [99]-[102]. The use of deep neural networks (DNNs) in neuroscience has created a heated debate about their scientific value and has spurred a discussion whether they are only valuable as predictive tools or might also offer helpful explanations of phenomena [103]. Cichy \& Kaiser (2019) establish three different perspectives from which DNNs have explanatory power: (i) they provide teleological explanations; (ii) despite their deceptive appearance, they provide the same explanations as traditional mathematical theoretical models; and (iii) owing to their complexity, they have strong potential for post hoc explanations.

Therefore, in addition to our DL network's ability to decode EEG data optimally and adequately, we designed the structure of the layers and their resulting weights in such a way that after the model learned the training data, we can gain insights into what the model deemed most predictive. Thus, we believe that the deliberate design of our layers allowed us to interpret the model's output in line with Cichy's \& Kaiser's (2019) arguments. The spectral filters embedded in our DL network provide insights into the neural frequencies most predictive of value. The spatial filters demonstrate the spatial distribution of the importance of each frequency, and the attention layers provide information regarding the critical timepoints for prediction. All these increase our understanding of the neural representation of value and its prediction in general.

\subsubsection{Spectral Filters and Spatial Filters}

Thanks to the network's architecture, we were able to interpret the first layer as bandpass frequency filters and the following convolution as band-specific spatial filters. Thus, we can observe the network's learned parameters in these layers and the process in which it extracted information. From these observations, we can learn what neural information did the network deem most predictive of WTP. In Fig. 5A, we plotted 3 example sinc functions that resulted from parameters in the first layer of the network. Below them, we show the results of an FFT analysis for these filters, exemplifying the frequency band that was passed in each of these filters. To understand which frequency bands the network utilized most for prediction, Fig. 5B shows all bandpass frequency ranges that the network provided (horizontal lines) and the overall probability of each frequency (grey histogram). As can be seen in Fig. 5B, we found that frequencies most prevalent in the first filters of the layer of the trained network (pink triangles and dotted pink lines) were within the beta band $12-30 \mathrm{~Hz}$, peaking at $14 \mathrm{~Hz}, 17-18 \mathrm{~Hz}, 20 \mathrm{~Hz}$, and $23 \mathrm{~Hz}$. Additionally, several additional smaller peaks can be found in the delta band (at 2-4Hz) and the alpha band (at $8-12 \mathrm{~Hz}$ ).

Fig. 5: Bandpass filters. (A) Top - 3 examples of filters from the trained network; bottom - the Fourier transform of each filter. (B) Aggregation of all sinc bandpass filters from the network. Each horizontal line represents a single bandpass filter, such that the line starts and ends at the filter's cutoff frequencies. The histogram in opaque grey shows the overall proportion of each frequency across all filters.

In addition to learning which frequency bands were the most informative for the networks' predictions, each bandpass filter generated by the network has five unique convolutional "spatial" filters that integrate the information from the different electrodes for each timepoint separately. We plotted these weights on topographic maps to illustrate how each spectral map, resulting from a bandpass filter, could be combined in various ways with respect to the electrode dimension. Fig. 6A shows these five maps for the three bandpass filters shown in Fig. 5A. They show that while some maps may be redundant and include similar weights (like in column number 3 - row 1 and 4 , or in column 2 - row 4 and 5), others may represent varied and even opposite integrations (as in column 1). Importantly, comparing these results from the individual spectral and spatial filters found in our study 
to EEG results found in previous literature would be amiss. We can see that out of all filters that the network generated, some frequencies and electrodes correspond with results from previous studies, while others do not. This provides further evidence that, while literature-based manual feature extraction could provide predictive information and meaningful insights, there could still be vast possible features that contain critical information that researchers could be unable to find through manual exploration.

Thus, as in Fig. 6B, we opted for an overall interpretation of these filters, by averaging the spatial weights within each of the standard EEG frequency band ranges. These demonstrate that, on average, the network utilized information from the frontal electrode FPZ the most, in all bands but Alpha. Interestingly, we can see a substantial positive contribution to prediction in frontal Delta, Beta, and Gamma, which have been established as associated with preferences [16]. Meanwhile, the Alpha band showed hemispheric asymmetry in the averaged weights, such that the right hemisphere was positively related to a positive prediction, while the left was negatively related. This brought to mind the EEG measure termed "Hemispheric Asymmetry", which was shown to reflect part of the valuation process, approach, or withdrawal [16]. Another EEG measure inspected in our previous paper [46], "Inter-Subject Correlation", is a measure of similarity between subjects, and therefore, we could not find means to relate it to the results in the current study.

Fig. 6: Spatial Frequency Illustration. (A) The interpolated spatial distribution of weights from the second layer is plotted as topographic maps. There are five spatial filters for each sinc bandpass filter, shown as five rows of maps in the figure. Each column represents a corresponding sinc filter, from left to right, as shown in Fig. 5A. (B) The average spatial weights per frequency band. Spatial weights from the second layer of the network were divided into their respective band (or nearest band), according to the bandpass filter to which they belong from the previous layer. Then, all spatial weights averaged within each band to yield eight final weights per frequency band.

\subsubsection{Attention Layer}

The attention layer provides a weight for each timepoint in the original EEG signal. These weights can be interpreted as the "importance" of each timepoint to the following prediction. Fig. 7 shows the distribution of weights, normalized between zero and one for better visualization, across all subjects and trials, and their median (represented as the blue line). Between timepoints -1 to 0 seconds (red area), subjects watched a black screen (the ITI); therefore, the importance is low for these timepoints. In timepoints $0.9-1.3$ seconds from stimulus onset (orange area), we can see an increase in importance, likely signifying initial processing of the stimulus's value. From timepoint 2.5 onwards, we can observe a substantial increase in importance (green area), showing that the most predictive information appears after internal deliberation and evaluation of the stimulus and right before the execution of the decision (which occurs from timepoint 3.5, when the stimulus is replaced by the BDM scale). The sharp increase towards the end of stimulus observation might be falsely interpreted as motor preparation towards moving the mouse cursor to either the left or right of the scale. However, to avert this critique, the mouse cursor's location on the scale was randomly initialized every trial, so subjects could not know in advance whether and how much they need to prepare to move the mouse right (high value) or left (low value).

Fig. 7: Distribution of weights from the attention layer. When the input reaches the attention layer, it is comprised of 75 timepoints (due to average pooling), each with its individual weight that is generated per input. We passed all samples through the final network and saved all weights. Thereon, we plotted a violin distribution of all weights per timepoint to generate this figure. Between timepoints -1 to 0 seconds (red area), subjects watched a black screen, between 0.9-1.3 seconds from stimulus onset (orange area), there was a slight increase in importance, and from timepoint 2.5 onwards, we can observe a substantial increase in importance (green area).

\subsection{Standard EEG Analysis \\ 3.3.1 ERP Analysis}

Inspecting Event-Related Potentials (ERP) is a common technique when analyzing EEG signals. In ERP analysis, we average the EEG recordings across different trials of each condition and examine if there are differences in the averaged electrical potentials between conditions. Differing neural responses could shed light on the neural mechanism that is at work in relation to the experimental manipulation between conditions. Therefore, after conducting the primary analysis using our DL network, we conducted several standard ERPbased analyses.

In a typical ERP design, there are multiple repetitions in each experimental condition in order to average out the noise in the signal. However, our experimental design was not a classic ERP design, as there were only three repetitions for each of the 72 products. Therefore, to have a better chance to observe distinct EEG components, and to gain more power in the statistical analysis, we created discrete conditions with a higher number of trials in each condition and averaged them, in order to clean the noise from the signal. So, we divided the data into four quartiles (serving as conditions) based on the WTP values. That is, we created four groups that correspond to the bottom $25 \%$ WTP values, $25 \%-50 \%$, 50\%-75\%, and $75 \%$ $100 \%$ of WTP values. We then averaged all the EEG activity from electrode FPZ for trials from each quartile across subjects and examined the signal within a 4.5 -seconds time window (3.5 seconds of product presentation plus 1 second from the previous ITI). We chose to examine the three frontal electrodes because we previously showed that they carry value-related information [46]. Moreover, because the correlations across the three frontal electrodes were very high $(\mathrm{r}=0.8-0.95$ across all subjects), it was enough to focus on the middle electrode, FPZ, as a representative for frontal activity.

As can be seen in Fig. 8A, three components of interest emerged. The N150 (negative potential after 150 milliseconds), P220 (positive potential after 220 milliseconds), and N300 (negative potential after 300 milliseconds) showed varying amplitudes across the WTP quartiles. The N150 has a lower amplitude for the highest and lowest WTP quartiles, perhaps measuring a response to the saliency of the products. We averaged the signal between 140 and 160 milliseconds per sample and found a significant difference between quartiles for this component $(\mathrm{F}(3)=5.12, \mathrm{p}<0.001$, one-way ANOVA). We conducted posthoc multiple comparison testing (Tukey), which revealed that the highest quartile had significantly lower N150 compared to the first $(p<0.001)$ and second $(\mathrm{p}<0.05)$ quartiles. The P220 and N300 components, in 
contrast, seem to exhibit sensitivity to value by having the lowest amplitude for the highest WTP quartile, perhaps encoding valuations. Again, we averaged the signal between 210 to 230 milliseconds and 290 to 310 milliseconds and found a significant difference between quartiles for these components as well (P220: $\mathrm{F}(3)=10.12$, $\mathrm{p}<0.001 ; \mathrm{N} 300$ : $\mathrm{F}(3)=$ $9.58, \mathrm{p}<0.001$, one way ANOVA). The same post-hoc analysis revealed that the $4^{\text {th }}$ quartile was significantly lower than all other quartiles in both components ( $p<0.001$ in all cases). We also broke up the analysis and examined the activity for every 0.5 seconds, starting at 0.5 seconds from stimulus onset, up to 3.5 seconds from the onset. However, these timepoints did not yield significant results.

However, note that many other characteristics of the signal could be different between the quartiles, in addition to what we captured by the three components identified (N150, P220, and N300). First, we demonstrated in the subsection "WTP Prediction" that predictions based on manually extracted features, including these components, were inferior to the automatic extraction by the neural network, aiding the conclusion that there were likely additional value-related signal characteristics that the DLN found than those captured by these components. For instance, as is common in the field, we manually and arbitrarily (by "visually inspecting the data") decided in which time window to average the signal and on which to conduct our statistical analyses. There are endless possibilities for manually choosing the onset and offset timepoints, and the window size for the analysis. It is very hard to know, a-priori, what would be the best combination of time window parameters to use. Moreover, any post-decision could lead to an inflated type one error and overfitting the data. To further highlight this problem, we repeated the exact same analysis for electrodes F7 and $\mathrm{Cz}$ (see Fig. 8B and Fig. 8C, respectively). Even without explicitly conducting a formal statistical analysis, it is clear that in these electrodes, the ordering of the quartiles is different for the three ERP components mentioned above. It might be that we could find in electrodes F7 and Cz other components, which would be significantly different between the WTP quartiles in a meaningful manner (either ascending or descending as a function of WTP values). However, finding these components could mean that we would need to examine endless combinations of time window parameters. This further strengthens the need for automatic feature extraction that would be sensitive to various time points, window sizes, and electrodes, as we incorporated in our deep learning network. We suggest that this manual procedure is most likely suboptimal for preference prediction compared to deep learning.

Fig. 8: Average ERP per quartile of WTP values. EEG recordings of electrode FPz (A), F7 (B), and Cz (C), after averaging for each WTP quartile, across subjects and products. The product image appeared at time zero, marking the start of a trial. Opaque bounds around a line signify the SEM at each data point. Cleary, each electrode recorded different responses and required diverse feature extraction procedures, further demonstrating the need for optimized and automatic feature extraction. The shaded areas mark timepoints where no difference between WTP quartiles was found. Dotted lines indicate the 150, 220, and 300-millisecond mark, respectively.

\subsubsection{Spectral Analysis}

The components derived from the event-related potentials often do not reveal the complete story, as they only expose task-relevant fluctuations in the time domain. Therefore, another popular technique for analyzing the EEG signal is to use spectral decomposition, which adds another perspective of analysis. Thus, we conducted a spectral analysis (as detailed in the methods section) and arranged the spectrograms according to the WTP quartiles. As a first-order analysis, similar to what we have shown in our previous studies [24], [46], we wanted to examine if there is a difference between the spectrograms of the top and bottom WTP quartiles and whether this difference was more pronounced than the difference between the two middle quartiles. Thus, we averaged the spectrogram within each quartile and subtracted the top quartile average spectrogram from the bottom quartile average (Fig. 9A), and from the third quartile average spectrogram, we subtracted the second quartile's average spectrogram (Fig. 9B).

As can be seen in Fig. 9A, there is a substantial difference between the top and bottom average spectrograms at the lower frequency range $(0.5-10 \mathrm{~Hz})$, around 0.5 seconds after the stimulus has appeared. This is indicated by the pink circle around a large dark blue dip at that timepoint, which reached a nearly -0.2 difference in log PSD. Importantly, additional frequencies at varied timepoints differed between high and low WTP values, as indicated by the dark red and blue colors, which represent a $\log$ difference of nearly 0.1 and -0.2 PSD, respectively.

The fact that there are numerous time points and frequencies that are significantly different between the top and bottom quartiles strengthens our notion that it would be futile to attempt to identify the most statistically relevant timepoints and frequencies, which would undoubtedly result in false positives even after correction for multiple comparisons. This suggests that the subjective value represented in the frequency domain is very complex. Trying to quantify subjective value representation into several features with any feature selection technique would probably be suboptimal and less generalizable. This serves as another testament as to why we advocate extracting features from the EEG signal automatically. The manual alternative, laboriously handpicking specific frequencies and timepoints, or aggregating frequency ranges to frequency bands (Alpha, Delta, etc.) or across timepoints, is suboptimal and involves information loss, as we demonstrated in the previous section based on the network prediction results.

Fig. 9: Spectrogram Differences for WTP quartiles. (A) The difference between the average spectrogram of the top and bottom average spectrogram quartiles. (B) The difference between the average spectrogram of the mid-high and mid-low average spectrogram quartiles. The product image appeared at time zero, marking the start of a trial. The dashed pink circle surrounded a substantial difference in the log PSDs.

\section{Discussion}

In this study, we showed that using a state-of-the-art DL network, it is possible to predict subjects' WTP values of products based on EEG recorded from the same subjects while they observed an image of the product. The unique network architecture, specially geared for EEG decoding and interpretation, alongside the meta-processes we used, reached $75.09 \%$ accuracy predicting above and below the median WTP value, with 0.832 AUC. The network also achieved 0.276 RMSE 
when predicting the continuous WTP values. In all cases, our network was better than other deep learning networks, exceeded results from a machine learning approach that utilized manual feature extraction, and surpassed random results (shuffled labels) by a large margin.

These results strengthen our notion that a DL framework approach, where the network itself determines the most relevant features and their appropriate weightings, is better than an approach where one needs to determine, a-priori, the exact features that serve as input data to the model. Because there are endless possibilities and degrees of freedom to this process, we strongly suggest moving beyond manual feature extraction towards automated feature extraction based on deep learning approaches.

Importantly, we showed that prediction scores were dependent on the extremity of WTP values. That is, prediction scores were superior for the prediction of the most extreme WTP values (0-20\% / 80-100\%) and decreased as WTP values became closer to each other. This reflects the notion of "neural distance" that was also found in previous studies, which states that as the distance between preferences increase, so does their neural representation, and prediction becomes better [24], [46], [96]

We conducted several vital analyses of the behavioral results to ensure that the BDM task was a close proxy for the subjective values that subjects had for the products and that we predicted these subjective values and not product identity. First, across subjects, there was a wide distribution of bids for each product, indicating a variety of preferences over the offered products between subjects. Only five products were overly disliked by subjects, with average bids under 10 NIS, while only five products were highly preferred, with average bids over 30 NIS. Therefore, $86 \%$ of products ranged within 10-30 NIS in their average bid across subjects. Second, we used a large number of products (72), which further reduced the probability that the network could identify neural information related to the product stimuli when predicting WTP, as WTP values would have to align similarly across all products for all subjects. Third, attempting to predict WTP based on product identities resulted in a prediction accuracy that was only slightly above shuffled data. Lastly, subjects demonstrated broadly consistent bids for each product across blocks, as demonstrated by high correlations between the bids across each pair of blocks. This suggests that subjects' bids were stable, and therefore, we could use them as a reliable representation of subjective value, and hence, as stable prediction labels.

We also analyzed our EEG data using standard approaches commonly used in the literature. We manually extracted features from specific time points and pre-defined frequency bands to look for components that could help predict WTP values. We wanted to show that this manual feature extraction and inspection, while it may lead to insightful observations, is very arbitrary and yields suboptimal predictions.

First, we conducted an event-related analysis. Based on this analysis, we found several interesting timepoints in the EEG time-series, averaged across all subjects and trials for each quartile of WTP. The average signal for the highest WTP quartile was lower than other quartiles on the 150-millisecond negative peak, 220-millisecond positive peak, and 300milliseconds negative peak. Event-related components within this time range were found to be related to value in various studies, such as the N200 [24], [104]-[106]. Finally, the average signal of the lowest quartile of WTP values showed the highest increase in voltage after the 300-millisecond mark, which could be related to the P300 component, that has been shown to be involved in valuation and decision making (Holroyd, Krigolson, \& Lee, 2011; Mushtaq, Wilkie, Mon-Williams, \& Schaefer, 2016; Proudfit, 2015; Hajcak, Holroyd, Moser, \& Simons, 2005; Hajcak, Moser, Holroyd, \& Simons, 2007; Luu, Shane, Pratt, \& Tucker, 2009; Sato et al., 2005; Toyomaki \& Murohashi, 2005; Yeung, Holroyd, \& Cohen, 2005; Yeung \& Sanfey, 2004; Gehring \& Willoughby, 2002; Holroyd \& Coles, 2002; Miltner, Braun, \& Coles, 1997; Yeung \& Sanfey, 2004). Importantly, by visually inspecting the EEG times-series, averaged by WTP quartiles, we showed that different electrodes produced various components with diverse relationships to value.

The multitude of optional components to choose from, which depend on varying scalp locations and varying timepoints, makes it very unlikely that a manual approach to feature extraction would allow for an accurate and generalizable model. Critically, we could not have known in advance to take these precise components, as we only found them through "peeking" into our prediction labels. This means that even if these features would contribute to WTP prediction, as they likely would in our case (since they fitted our data), they would not necessarily be found as the most contributive in another dataset. Moreover, when we added these components for the prediction of WTP using ML models, it still resulted in far worse predictions than the DL approaches. Hence, the large variety of optional ERP components, the lack of explicit directions on how to choose among them, and the inferior results from the ML prediction approach using manual extraction further substantiate our claim that automatic feature extraction should surpass any attempt to capture value-related information hidden in the EEG signal manually.

In addition to maximizing our deep learning network's prediction accuracy, we also engineered it so that we could interpret the results, and therefore, learn from it regarding the neural representation of value. Based on examining the output of the first and second layer of the network, we found evidence that some EEG features previously shown to be related to preference prediction also contributed to prediction in our network. Specifically, based on the output of the network's first layer, we learned that the beta band was most important for the prediction of WTP, in correspondence with previous research [37], [121], while alpha and delta bands also contributed substantially [22], [28], [121]-[123]. Besides, by averaging the spatial weights from the second layer within each of the standard frequency bands, we found that the network relied mainly on the frontal electrode FPZ for its prediction, in correspondence with previous research which related frontal cortical activity with value [16]. Meanwhile, in the alpha band, we found evidence for hemispheric asymmetry, which is commonly considered as a measure of engagement, or approach/avoidance [21], [27], [129], [28], [29], [36], [124]-[128].

However, the frequency bands we identified in the first layer and the scalp distribution of each of the spectral maps from the second layer were both highly varied. As in the case of the event-related analysis, this demonstrates that it is near impossible to manually identify the most predictive 
frequencies and their most fitting scalp distribution. Unfortunately, this is often the approach in many EEG studies. It is very unlikely that researchers could estimate which frequencies to extract and how to combine their scalp information. We propose that using a DL framework approach provides an automated and better solution to address this. Moreover, studies often combine information from electrodes in a single manner, such as averaging or conducting an Independent Component Analysis (ICA), and combine the electrodes for all frequency ranges using only one method [18]. In contrast, our network architecture defined several unique electrode combinations for each bandpass filter separately, enabling substantially larger expressiveness in extracting features from the signal. The network produced several sets of weights for the electrode dimensions for each spectral map produced by a bandpass filter. Some were unique while others were redundant, thus enabling varied ways to combine the electrodes' information that were specialized and independent per bandpass filter, without the need for relying on the popular frequency bands (alpha, beta, gamma, delta, and theta).

Finally, the attention layer was able to expose the timepoints that were most important for preference prediction. Averaging the attention weights across all samples revealed an uplift in predictive information one second after stimulus onset. This possibly reflected early processing of the stimulus's value without converging to a final valuation yet. Thereon, the averaged weights increased substantially until reaching their highest just before decision execution. This pattern might be interpreted as being related to the motor preparation before executing the decision - reporting a WTP value on the sliding scale - and not to value per se. However, we do not think this is the case because we randomized the initial location of the marker on the scale, so subjects could not have known in advance whether and by how much they would need to move the mouse cursor to the left or the right to reach their desired bid. Therefore, we propose that the rise of importance towards decision execution demonstrated that subjects thought about and determined their bid gradually while they watched the product, and that it peaked just before they had to report it. This process resembles the well-known evidence accumulation models for describing choice [130][132].

There are several avenues of research that could expand this study. First, our predictions were limited to preferences elicited immediately after recording the EEG response, but future studies could examine the ability to predict lasting preferences elicited an hour or a week after EEG was recorded. The consumer neuroscience field and the neuromarketing industry are likely interested in predicting durable and stable preferences, so this study can provide the basis for researchers to examine to what degree these long-term preferences can be predicted. Additionally, it is often desired to predict a product's success in the general population rather than predicting individual subjective valuations. Future research could also inspect whether EEG recordings can predict preferences over products in the general population, as estimated via general sales, YouTube measures, or other accepted metrics. Finally, researchers could build upon these findings and examine whether they could predict preferences over products from EEG recorded while subjects observe video ads instead of product images. Demonstrating that preferences could still be predicted based on more complex and ecological stimuli would provide neuromarketers with valuable evidence of the EEG signal's capability as a tool to assess ad effectiveness and predict marketing gains.

\subsection{Conclusion}

We were able to show that a deep-learning approach with carefully constructed architecture could be ideal for EEGbased preference prediction, surpassing both manual approaches and generic DL frameworks. Our DL network generated features to a degree of complexity, spatially, and spectrally that could not have been achieved through manual inspection and feature extraction. Importantly, our datadriven approach shed light on some aspects of the valuations process, as reflected in the EEG signal, that were not previously investigated or were unlikely to be included in a prediction model that uses manual feature extraction. The network employed an abundance of frequencies, spatial distributions, and timepoints, focusing mainly on the aftermost timepoints. Thus, neuromarketing practitioners can utilize our DL approach to enhance their attempts to predict preferences based on EEG data, regardless of the marketing stimuli. Meanwhile, EEG and decision-making researchers can employ this framework learn more about the valuation process by investigating the network's resulting parameters and exploring which neural features the network deemed most essential for prediction.

\section{Competing financial interests}

The authors declare no competing financial interests.

\section{Acknowledgements}

We want to thank all Dino Levy's lab members for their help and guidance in many discussions. Thanks to Matan Cohen and Yaron Geffen for their thoughts and advice on the paper, and their review of existing DL frameworks. DJL thanks the Israel Science Foundation (grant \#954/17) for their support of this study

\section{Author contributions statement}

AH performed the data analysis and visualizations, oversaw the data collection, and created the prediction models. IG contributed to the modeling, designed the model optimizations, and wrote the related sections. AH and DJL designed the experiment and wrote the manuscript. SY conducted the experimental procedure. All authors read and approved the final manuscript.

\section{References}

[1] D. Ariely and G. S. Berns, "Neuromarketing: the hope and hype of neuroimaging in business," Nat. Rev. Neurosci., vol. 11, no. 4, pp. 284 292, Apr. 2010.

[2] V. Fortunato, J. Giraldi, and J. Oliveira, "A Review of Studies on Neuromarketing: Practical Results, Techniques, Contributions and Limitations," J. Manag. Res., vol. 6, no. 2, p. 201, 2014.

[3] A. Javor, M. Koller, N. Lee, L. Chamberlain, and G. Ransmayr, "Neuromarketing and consumer neuroscience: Contributions to neurology," BMC Neurol., vol. 13, pp. 1-12, 2013.

[4] A. Genevsky, C. Yoon, and B. Knutson, "When brain beats behavior: Neuroforecasting crowdfunding outcomes," J. Neurosci., pp. 163316, 2017.

[5] H. Plassmann, V. Venkatraman, S. Huettel, and C. Yoon, "Consumer Neuroscience: Applications, Challenges, and Possible Solutions," J. Mark. Res., vol. 52, no. 4, pp. 427-435, Aug. 2015.

[6] U. R. Karmarkar and H. Plassmann, “Consumer Neuroscience: Past, 
Present and Future," Organ. Res. Methods, p. 109442811773059, 2017.

[7] N. Lee, L. Brandes, L. Chamberlain, and C. Senior, "This is your brain on neuromarketing: reflections on a decade of research," J. Mark. Manag., vol. 33, no. 11-12, pp. 878-892, 2017.

[8] T. Schneider and S. Woolgar, "Neuromarketing in the making: Enactment and reflexive entanglement in an emerging field," Biosocieties, vol. 10, no. 4, 2015.

[9] A. Smidts et al., "Advancing consumer neuroscience," Mark. Lett., 2014.

[10] J. M. Harris, J. Ciorciari, and J. Gountas, "Consumer neuroscience for marketing researchers," J. Consum. Behav., vol. 17, no. 3, pp. 239-252, 2018.

[11] M. Hsu, "Neuromarketing: Inside the Mind of the Consumer," Calif. Manage. Rev., vol. 59, no. 4, pp. 5-22, 2017.

[12] M. Hsu and C. Yoon, "The neuroscience of consumer choice," Current Opinion in Behavioral Sciences, vol. 5. 2015.

[13] U. R. Karmarkar and C. Yoon, "Consumer neuroscience: Advances in understanding consumer psychology," Curr. Opin. Psychol., vol. 10, pp. 160-165, 2016.

[14] M. H. Lin, S. N. N. Cross, W. J. Jones, and T. L. Childers, "Applying EEG in consumer neuroscience," Eur. J. Mark., vol. 52, no. 1-2, pp. 66-91, 2018.

[15] P. Cherubino et al., "Consumer behaviour through the eyes of neurophysiological measures: State-of-the-art and future trends," Comput. Intell. Neurosci., vol. 2019, 2019.

[16] A. Hakim and D. J. Levy, "A gateway to consumers' minds: Achievements, caveats, and prospects of electroencephalographybased prediction in neuromarketing," Wiley Interdisciplinary Reviews: Cognitive Science, vol. 10, no. 2. pp. 1-21, 2019.

[17] L. F. Haas, "Hans Berger (1873-1941), Richard Caton (1842-1926), and electroencephalography.," J. Neurol. Neurosurg. Psychiatry, vol. 74, no. 1, p. 9, Jan. 2003.

[18] S. J. Luck, An introduction to the event-related potential technique, Second edi. MIT Press, 2014.

[19] L. Fuentemilla, D. Cucurell, J. Marco-Pallarés, M. Guitart-Masip, J. Morís, and A. Rodríguez-Fornells, "Electrophysiological correlates of anticipating improbable but desired events," Neuroimage, vol. 78, pp. 135-144, 2013.

[20] R. San Martin, L. G. Appelbaum, J. M. Pearson, S. A. Huettel, and M. G. Woldorff, "Rapid Brain Responses Independently Predict Gain Maximization and Loss Minimization during Economic Decision Making," J. Neurosci., vol. 33, no. 16, pp. 7011-7019, 2013.

[21] S. K. Sutton and R. J. Davidson, "Prefrontal brain electrical asymmetry predicts the evaluation of affective stimuli," Neuropsychologia, vol. 38, no. 13, pp. 1723-1733, Dec. 2000.

[22] R. N. Khushaba, C. Wise, S. Kodagoda, J. Louviere, B. E. Kahn, and C. Townsend, "Consumer neuroscience: Assessing the brain response to marketing stimuli using electroencephalogram (EEG) and eye tracking," Expert Syst. Appl., vol. 40, no. 9, pp. 3803-3812, 2013.

[23] J. P. Dmochowski, P. Sajda, J. Dias, and L. C. Parra, "Correlated Components of Ongoing EEG Point to Emotionally Laden Attention - A Possible Marker of Engagement?," Front. Hum. Neurosci., vol. 6, no. May, pp. 1-9, 2012.

[24] A. Telpaz, R. Webb, and D. J. Levy, "Using EEG to Predict Consumers' Future Choices," J. Mark. Res., vol. 52, no. 4, pp. 511-529, 2015.

[25] M. Yadava, P. Kumar, R. Saini, P. P. Roy, and D. Prosad Dogra, "Analysis of EEG signals and its application to neuromarketing," Multimed. Tools Appl., vol. 76, no. 18, pp. 19087-19111, 2017.

[26] W. Kong, X. Zhao, S. Hu, G. Vecchiato, and F. Babiloni, "Electronic evaluation for video commercials by impression index," Cogn.
Neurodyn., vol. 7, no. 6, pp. 531-535, 2013.

[27] G. Vecchiato et al., "Spectral EEG frontal asymmetries correlate with the experienced pleasantness of TV commercial advertisements," Med. Biol. Eng. Comput., vol. 49, no. 5, pp. 579-583, 2011.

[28] N. Ravaja, O. Somervuori, and M. Salminen, "Predicting purchase decision: The role of hemispheric asymmetry over the frontal cortex.," J. Neurosci. Psychol. Econ., vol. 6, no. 1, pp. 1-13, 2013.

[29] T. Z. Ramsøy, M. Skov, M. K. Christensen, and C. Stahlhut, "Frontal brain asymmetry and willingness to pay," Front. Neurosci., vol. 12, no. MAR, 2018.

[30] Z. Wei, C. Wu, X. Wang, A. Supratak, P. Wang, and Y. Guo, “Using support vector machine on EEG for advertisement impact assessment," Front. Neurosci., vol. 12, no. MAR, 2018.

[31] S. Kumar, M. Yadava, and P. P. Roy, "Fusion of EEG response and sentiment analysis of products review to predict customer satisfaction," Inf. Fusion, vol. 52, no. August 2017, pp. 41-52, 2019.

[32] P. Golnar-nik, S. Farashi, and M. Safari, "Physiology \& Behavior The application of EEG power for the prediction and interpretation of consumer decision-making: A neuromarketing study," Physiol. Behav., vol. 207, no. August 2018, pp. 90-98, 2019.

[33] P. Pandey, R. Swarnkar, S. Kakaria, and K. P. Miyapuram, “Understanding Consumer Preferences for Movie Trailers from EEG using Machine Learning," Jul. 2020.

[34] Y. Si et al., "Predicting individual decision-making responses based on single-trial EEG," Neuroimage, vol. 206, p. 116333, Feb. 2020.

[35] N. Alnuman, S. Al-Nasser, and O. Yasin, "Classification of Products Preference from EEG Signals using SVM Classifier," 2020, pp. 174179.

[36] V. Venkatraman et al., "Predicting Advertising Success Beyond Traditional Measures: New Insights from Neurophysiological Methods and Market Response Modeling," J. Mark. Res., vol. 52, no. 4, pp. 436-452, 2015.

[37] M. A. S. Boksem and A. Smidts, "Brain Responses to Movie Trailers Predict Individual Preferences for Movies and Their PopulationWide Commercial Success," J. Mark. Res., vol. 52, no. 4, pp. 482-492, 2015.

[38] J. P. Dmochowski, M. A. Bezdek, B. P. Abelson, J. S. Johnson, E. H. Schumacher, and L. C. Parra, "Audience preferences are predicted by temporal reliability of neural processing," Nat. Commun., vol. 5, pp. $1-9,2014$.

[39] S. B. Barnett and M. Cerf, "A ticket for your thoughts: Method for predicting content recall and sales using neural similarity of moviegoers," J. Consum. Res., vol. 44, no. 1, pp. 160-181, 2017.

[40] J. Guixeres et al., "Consumer neuroscience-based metrics predict recall, liking and viewing rates in online advertising," Front. Psychol., vol. 8, no. OCT, pp. 1-14, 2017.

[41] C. Christoforou, T. C. Papadopoulos, F. Constantinidou, and M. Theodorou, "Your Brain on the Movies: A Computational Approach for Predicting Box-office Performance from Viewer's Brain Responses to Movie Trailers," Front. Neuroinform., vol. 11, p. 72, Dec. 2017.

[42] E. Eijlers, M. A. S. Boksem, and A. Smidts, "Measuring Neural Arousal for Advertisements and Its Relationship With Advertising Success," Front. Neurosci., vol. 14, no. July, pp. 1-12, 2020.

[43] A. Y. Shestyuk, K. Kasinathan, V. Karapoondinott, R. T. Knight, and R. Gurumoorthy, "Individual EEG measures of attention, memory, and motivation predict population level TV viewership and Twitter engagement," PLoS One, vol. 14, no. 3, p. e0214507, Mar. 2019.

[44] D. Baldo, H. Parikh, Y. Piu, and K.-M. Müller, "Brain Waves Predict Success of New Fashion Products: A Practical Application for the Footwear Retailing Industry," J. Creat. Value, vol. 1, no. 1, pp. 61-71, 2015. 
[45] M. Murugappan, S. Murugappan, Balaganapathy, and C. Gerard, "Wireless EEG signals based Neuromarketing system using Fast Fourier Transform (FFT)," 2014 IEEE 10th Int. Colloq. Signal Process. its Appl., pp. 25-30, 2014.

[46] A. Hakim, S. Klorfeld, T. Sela, D. Friedman, M. Shabat-Simon, and D. J. Levy, "Machines learn neuromarketing: Improving preference prediction from self-reports using multiple EEG measures and machine learning," Int. J. Res. Mark., Dec. 2021.

[47] R. A. Poldrack et al., "Scanning the horizon: Towards transparent and reproducible neuroimaging research," Nat. Rev. Neurosci., vol. 18, no. 2, pp. 115-126, Feb. 2017.

[48] Y. LeCun, Y. Bengio, and G. Hinton, "Deep learning," Nature, vol. 521, no. 7553, pp. 436-444, May 2015.

[49] X. Gu et al., "EEG-based Brain-Computer Interfaces (BCIs): A Survey of Recent Studies on Signal Sensing Technologies and Computational Intelligence Approaches and their Applications," 2020.

[50] A. Khosla, P. Khandnor, and T. Chand, "A comparative analysis of signal processing and classification methods for different applications based on EEG signals," Integr. Med. Res., 2020.

[51] A. Antoniades, L. Spyrou, C. C. Took, and S. Sanei, "Deep learning for epileptic intracranial EEG data," in IEEE International Workshop on Machine Learning for Signal Processing, MLSP, 2016, vol. 2016-Novem, pp. 1-6.

[52] J. Liang, R. Lu, C. Zhang, and F. Wang, "Predicting Seizures from Electroencephalography Recordings: A Knowledge Transfer Strategy," in 2016 IEEE International Conference on Healthcare Informatics (ICHI), 2016, pp. 184-191.

[53] P. Mirowski, D. Madhavan, Y. LeCun, and R. Kuzniecky, "Classification of patterns of EEG synchronization for seizure prediction," Clin. Neurophysiol., vol. 120, no. 11, pp. 1927-1940, Nov. 2009.

[54] P. Thodoroff, J. Pineau, and A. Lim, "Learning Robust Features using Deep Learning for Automatic Seizure Detection," 2016.

[55] A. Page, C. Shea, and T. Mohsenin, "Wearable seizure detection using convolutional neural networks with transfer learning," in 2016 IEEE International Symposium on Circuits and Systems (ISCAS), 2016, pp. 1086-1089.

[56] M.-P. Hosseini, H. Soltanian-Zadeh, K. Elisevich, and D. Pompili, "Cloud-based deep learning of big EEG data for epileptic seizure prediction," 2016 IEEE Glob. Conf. Signal Inf. Process. (GlobalSIP, pp. 1151-1155, 2016.

[57] U. R. Acharya, S. L. Oh, Y. Hagiwara, J. H. Tan, and H. Adeli, “Deep convolutional neural network for the automated detection and diagnosis of seizure using EEG signals," Comput. Biol. Med., vol. 100, pp. 270-278, Sep. 2018.

[58] M. Golmohammadi, V. Shah, I. Obeid, and J. Picone, “Deep Learning Approaches for Automated Seizure Detection from Scalp Electroencephalograms," 2017.

[59] S. Stober, D. J. Cameron, and J. A. Grahn, “Using Convolutional Neural Networks to Recognize Rhythm [o Electroencephalography Recordings." pp. 1449-1457, 2014.

[60] S. Stober, A. Sternin, A. M. Owen, and J. A. Grahn, “Deep Feature Learning for EEG Recordings," 2015.

[61] H. Cecotti and A. Graser, "Convolutional Neural Networks for P300 Detection with Application to Brain-Computer Interfaces," IEEE Trans. Pattern Anal. Mach. Intell., vol. 33, no. 3, pp. 433-445, Mar. 2011.

[62] R. Manor and A. B. Geva, "Convolutional Neural Network for MultiCategory Rapid Serial Visual Presentation BCI," Front. Comput. Neurosci., vol. 9, p. 146, Dec. 2015.

[63] J. Shamwell, H. Lee, H. Kwon, A. R. Marathe, V. Lawhern, and W. Nothwang, "Single-trial EEG RSVP classification using convolutional neural networks," 2016, vol. 9836, p. 983622.

[64] H. Cecotti, M. P. Eckstein, and B. Giesbrecht, "Single-Trial Classification of Event-Related Potentials in Rapid Serial Visual Presentation Tasks Using Supervised Spatial Filtering," IEEE Trans. Neural Networks Learn. Syst., vol. 25, no. 11, pp. 2030-2042, Nov. 2014.

[65] P. Bashivan, I. Rish, M. Yeasin, and N. Codella, "Learning Representations from EEG with Deep Recurrent-Convolutional Neural Networks," pp. 1-15, 2015.

[66] M. A. Almogbel, A. H. Dang, and W. Kameyama, "Cognitive Workload Detection from Raw EEG-Signals of Vehicle Driver using Deep Learning," Int. Conf. Adv. Commun. Technol. ICACT, vol. 2019Febru, no. 6, pp. 1167-1172, 2019.

[67] A. I. Humayun, A. S. Sushmit, T. Hasan, and M. I. H. Bhuiyan, “EndTo-end sleep staging with raw single channel EEG using deep residual convnets," in 2019 IEEE EMBS International Conference on Biomedical and Health Informatics, BHI 2019 - Proceedings, 2019, pp. 15.

[68] A. Supratak, H. Dong, C. Wu, and Y. Guo, "DeepSleepNet: A model for automatic sleep stage scoring based on raw single-channel EEG," IEEE Trans. Neural Syst. Rehabil. Eng., vol. 25, no. 11, pp. 1998-2008, 2017.

[69] A. Sors, S. Bonnet, S. Mirek, L. Vercueil, and J. F. Payen, "A convolutional neural network for sleep stage scoring from raw single-channel EEG," Biomed. Signal Process. Control, vol. 42, pp. 107114, 2018.

[70] Y. Yuan et al., "A hybrid self-attention deep learning framework for multivariate sleep stage classification," BMC Bioinformatics, vol. 20, no. Suppl 16, pp. 1-10, 2019.

[71] N. Michielli, U. R. Acharya, and F. Molinari, “Cascaded LSTM recurrent neural network for automated sleep stage classification using single-channel EEG signals," Comput. Biol. Med., vol. 106, pp. 71-81, Mar. 2019.

[72] E. Qazi, M. Hussain, and H. Aboalsamh, "Automatic Emotion Recognition ( AER ) System based on Two- Level Ensemble of Lightweight Deep CNN Models," no. 2, 2019.

[73] W. L. Zheng and B. L. Lu, "Investigating Critical Frequency Bands and Channels for EEG-Based Emotion Recognition with Deep Neural Networks," IEEE Trans. Auton. Ment. Dev., vol. 7, no. 3, pp. 162-175, Sep. 2015.

[74] J. Teo, L. H. Chew, J. T. Chia, and J. Mountstephens, “Classification of affective states via EEG and deep learning," Int. J. Adv. Comput. Sci. Appl., vol. 9, no. 5, pp. 132-142, 2018.

[75] C. Li, Z. Bao, L. Li, and Z. Zhao, "Exploring temporal representations by leveraging attention-based bidirectional LSTM-RNNs for multimodal emotion recognition," Inf. Process. Manag., vol. 57, no. 3, p. 102185, 2020.

[76] S. Jirayucharoensak, S. Pan-Ngum, P. Israsena, S. Jirayucharoensak, S. Pan-Ngum, and P. Israsena, "EEG-Based Emotion Recognition Using Deep Learning Network with Principal Component Based Covariate Shift Adaptation, EEG-Based Emotion Recognition Using Deep Learning Network with Principal Component Based Covariate Shift Adaptation," vol. 2014, 2014, p. e627892, 2014.

[77] H. Zeng, Z. Wu, J. Zhang, C. Yang, H. Zhang, and G. Dai, “brain sciences EEG Emotion Classification Using an Improved SincNetBased Deep Learning Model," 2019.

[78] B. H. Kim and S. Jo, "Deep Physiological Affect Network for the Recognition of Human Emotions," IEEE Trans. Affect. Comput., vol. 14, no. 8, 2018.

[79] J. X. Chen, P. W. Zhang, Z. J. Mao, Y. F. Huang, D. M. Jiang, and Y. N. Zhang, "Accurate EEG-Based Emotion Recognition on Combined Features Using Deep Convolutional Neural Networks," IEEE Access, vol. 7, pp. 44317-44328, 2019. 
[80] S. Nakagome, T. P. Luu, Y. He, A. S. Ravindran, and J. L. ContrerasVidal, "An empirical comparison of neural networks and machine learning algorithms for EEG gait decoding," Sci. Rep., vol. 10, no. 1, p. 4372,2020 .

[81] A. Jalaly, H. J. Bidgoly, and Z. Arezoumand, "A Survey on Methods and Challenges in EEG Based Authentication," Comput. Secur., p. 101788,2020

[82] R. T. Schirrmeister et al., "Deep learning with convolutional neural networks for EEG decoding and visualization," Hum. Brain Mapp., vol. 38, no. 11, pp. 5391-5420, 2017.

[83] Y. R. Tabar and U. Halici, "A novel deep learning approach for classification of EEG motor imagery signals," J. Neural Eng., vol. 14, no. 1, p. 016003 , Feb. 2017.

[84] X. An, D. Kuang, X. Guo, Y. Zhao, and L. He, "A Deep Learning Method for Classification of EEG Data Based on Motor Imagery," Intell. Comput. Bioinforma., pp. 203-210, 2014.

[85] S. Sakhavi, C. Guan, and S. Yan, "Parallel convolutional-linear neural network for motor imagery classification," in 2015 23rd European Signal Processing Conference (EUSIPCO), 2015, pp. 2736-2740.

[86] Z. Tayeb, J. Fedjaev, N. Ghaboosi, and C. Richter, "Validating Deep Neural Networks for Online Decoding of Motor Imagery Movements from EEG Signals," no. September, pp. 1-16, 2018.

[87] Z. Tang, C. Li, and S. Sun, "Single-trial EEG classification of motor imagery using deep convolutional neural networks," Optik (Stuttg)., vol. 130, 2017.

[88] B. E. Olivas-Padilla and M. I. Chacon-Murguia, "Classification of multiple motor imagery using deep convolutional neural networks and spatial filters," Appl. Soft Comput., 2018.

[89] J. Li and A. Cichocki, "Deep Learning of Multifractal Attributes from Motor Imagery Induced EEG," Neural Inf. Process., pp. 503-510, 2014.

[90] S. Sakhavi and C. Guan, "Convolutional neural network-based transfer learning and knowledge distillation using multi-subject data in motor imagery BCI," Int. IEEE/EMBS Conf. Neural Eng. NER, pp. 588-591, 2017.

[91] W. Qiao, "Deep Spatial-Temporal Neural Network for Classification of EEG-Based Motor Imagery," pp. 265-272, 2019.

[92] Y. Roy, H. Banville, I. Albuquerque, A. Gramfort, T. H. Falk, and J. Faubert, "Deep learning-based electroencephalography analysis: A systematic review," Journal of Neural Engineering, vol. 16, no. 5. 2019.

[93] G. M. Becker, M. H. Degroot, and J. Marschak, "Measuring utility by a single-response sequential method," Behav. Sci., vol. 9, no. 3, pp. 226-232, Jan. 1964.

[94] A. Delorme and S. Makeig, "EEGLAB: An open source toolbox for analysis of single-trial EEG dynamics including independent component analysis," J. Neurosci. Methods, vol. 134, no. 1, pp. 9-21, Mar. 2004.

[95] J. Bergstra, R. Bardenet, Y. Bengio, and B. Kégl, "Algorithms for Hyper-Parameter Optimization," 2011.

[96] I. Levy, S. C. Lazzaro, R. B. Rutledge, and P. W. Glimcher, "Choice from Non-Choice: Predicting Consumer Preferences from Blood Oxygenation Level-Dependent Signals Obtained during Passive Viewing," J. Neurosci., vol. 31, no. 1, pp. 118-125, 2011.

[97] T. Chen and C. Guestrin, "XGBoost: A scalable tree boosting system," in Proceedings of the ACM SIGKDD International Conference on Knowledge Discovery and Data Mining, 2016, vol. 13-17-August-2016, pp. 785-794.

[98] V. J. Lawhern, A. J. Solon, N. R. Waytowich, S. M. Gordon, C. P. Hung, and B. J. Lance, "EEGNet: A Compact Convolutional Network for EEG-based Brain-Computer Interfaces," pp. 1-30, 2016.

[99] F. K. Dosilovic, M. Brcic, and N. Hlupic, "Explainable artificial intelligence: A survey," 2018 41st Int. Conv. Inf. Commun. Technol. Electron. Microelectron. MIPRO 2018 - Proc., no. May, pp. 210-215,
2018.

[100]W. Samek, G. Montavon, A. Vedaldi, L. K. Hansen, and K.-R. Muller, Explainable AI: Interpreting, Explaining and Visualizing Deep Learning, vol. 11700. Cham: Springer International Publishing, 2019.

[101]N. Xie, G. Ras, M. van Gerven, and D. Doran, "Explainable deep learning: A field guide for the uninitiated," arXiv. arXiv, 29-Apr2020.

[102]W. Samek, T. Wiegand, and K. R. Müller, “Explainable artificial intelligence: Understanding, visualizing and interpreting deep learning models," arXiv. 2017

[103]R. M. Cichy and D. Kaiser, "Deep Neural Networks as Scientific Models," Trends in Cognitive Sciences, vol. 23, no. 4. Elsevier Ltd, pp. 305-317, 01-Apr-2019.

[104]N. Goto et al., "Neural signals of selective attention are modulated by subjective preferences and buying decisions in a virtual shopping task," Biol. Psychol., vol. 128, no. June, pp. 11-20, 2017.

[105]P. D. Gajewski, J. Drizinsky, J. Zulch, and M. Falkenstein, “ERP correlates of simulated purchase decisions," Front. Neurosci., vol. 10, no. AUG, pp. 1-13, 2016.

[106]J. R. Folstein and C. Van Petten, "Influence of cognitive control and mismatch on the N2 component of the ERP: A review," Psychophysiology, vol. 0, no. 0, pp. 070915195953001-???, Sep. 2007.

[107]F. Mushtaq, R. M. Wilkie, M. A. Mon-Williams, and A. Schaefer, "Randomised prior feedback modulates neural signals of outcome monitoring," Neuroimage, vol. 125, pp. 868-879, Jan. 2016.

[108]G. H. Proudfit, "The reward positivity: From basic research on reward to a biomarker for depression," Psychophysiology, vol. 52, no. 4, pp. 449-459, Apr. 2015.

[109]C. B. Holroyd, O. E. Krigolson, and S. Lee, "Reward positivity elicited by predictive cues," Neuroreport, vol. 22, no. 5, pp. 249-252, Mar. 2011.

[110]Z. Kardos, B. Tóth, R. Boha, B. File, and M. Molnár, “Age-dependent characteristics of feedback evaluation related to monetary gains and losses," Int. J. Psychophysiol., vol. 122, pp. 42-49, 2017.

[111]G. Hajcak, C. B. Holroyd, J. S. Moser, and R. F. Simons, "Brain potentials associated with expected and unexpected good and bad outcomes," Psychophysiology, vol. 42, no. 2, pp. 161-170, Mar. 2005.

[112]G. Hajcak, J. S. Moser, C. B. Holroyd, and R. F. Simons, “It's worse than you thought: The feedback negativity and violations of reward prediction in gambling tasks," Psychophysiology, vol. 44, no. 6, pp. 905-912, Nov. 2007.

[113]P. Luu, M. Shane, N. L. Pratt, and D. M. Tucker, "Corticolimbic mechanisms in the control of trial and error learning," Brain Res., vol. 1247, pp. 100-113, Jan. 2009.

[114]A. Sato et al., "Effects of value and reward magnitude on feedback negativity and P300," Neuroreport, vol. 16, no. 4, pp. 407-411, Mar. 2005.

[115]A. Toyomaki and H. Murohashi, "The ERPs to feedback indicating monetary loss and gain on the game of modified 'rock-paperscissors,"' Int. Congr. Ser., vol. 1278, pp. 381-384, Mar. 2005.

[116]N. Yeung and A. G. Sanfey, "Independent coding of reward magnitude and valence in the human brain.," J. Neurosci., vol. 24, no. 28, pp. 6258-64, Jul. 2004.

[117]N. Yeung, C. B. Holroyd, and J. D. Cohen, "ERP Correlates of Feedback and Reward Processing in the Presence and Absence of Response Choice," Cereb. Cortex, vol. 15, no. 5, pp. 535-544, May 2005.

[118]W. J. Gehring and A. R. Willoughby, "The medial frontal cortex and the rapid processing of monetary gains and losses.," Science, vol. 295, no. 5563, pp. 2279-82, Mar. 2002.

[119]C. B. Holroyd and M. G. H. Coles, "The neural basis of human error processing: Reinforcement learning, dopamine, and the error-related negativity.," Psychol. Rev., vol. 109, no. 4, pp. 679-709, Oct. 2002. 
[120]W. H. R. Miltner, C. H. Braun, and M. G. H. Coles, “Event-Related Brain Potentials Following Incorrect Feedback in a Time-Estimation Task: Evidence for a 'Generic' Neural System for Error Detection," J. Cogn. Neurosci., vol. 9, no. 6, pp. 788-798, Nov. 1997.

[121]S. Braeutigam, S. P. R. Rose, S. J. Swithenby, and T. Ambler, "The distributed neuronal systems supporting choice-making in real-life situations: Differences between men and women when choosing groceries detected using magnetoencephalography," Eur. J. Neurosci., vol. 20, no. 1, pp. 293-302, 2004.

[122]M. E. Smith and A. Gevins, "Attention and brain activity while watching television: Components of viewer engagement," Media Psychol., vol. 6, no. 3, pp. 285-305, 2004.

[123]L. Astolfi et al., "Neural basis for brain responses to TV commercials: A high-resolution EEG study," IEEE Trans. Neural Syst. Rehabil. Eng., vol. 16, no. 6, pp. 522-531, 2008.

[124]R. J. Davidson, "Anterior electrophysiological asymmetries, emotion, and depression: Conceptual and methodological conundrums," Psychophysiology, vol. 35, no. 5, pp. 607-614, 1998.

[125]R. Ohme, D. Reykowska, D. Wiener, and A. Choromanska, "Analysis of Neurophysiological Reactions to Advertising Stimuli by Means of EEG and Galvanic Skin Response Measures," J. Neurosci. Psychol. Econ., vol. 2, no. 1, pp. 21-31, 2009.

[126]R. Ohme, D. Reykowska, D. Wiener, and A. Choromanska,
"Application of frontal EEG asymmetry to advertising research," $J$. Econ. Psychol., vol. 31, no. 5, pp. 785-793, 2010.

[127]G. Cartocci et al., "Gender and Age Related Effects while Watching TV Advertisements: An EEG Study," Comput. Intell. Neurosci., vol. 2016, pp. 1-11, 2016.

[128]G. Vecchiato et al., "Changes in brain activity during the observation of TV commercials by using EEG, GSR and HR measurements," Brain Topogr., 2010.

[129]S. Koelstra et al., "DEAP: A database for emotion analysis; Using physiological signals," IEEE Trans. Affect. Comput., vol. 3, no. 1, pp. 18-31, 2012.

[130]I. Krajbich, C. Armel, and A. Rangel, "Visual fixations and the computation and comparison of value in simple choice," Nat. Neurosci., vol. 13, no. 10, pp. 1292-1298, Oct. 2010.

[131]R. Ratcliff, "A theory of memory retrieval," Psychol. Rev., vol. 85, no. 2, pp. 59-108, Mar. 1978.

[132]J. I. Gold and M. N. Shadlen, "The neural basis of decision making," Annual Review of Neuroscience, vol. 30. Annual Reviews, pp. 535-574, 28-Jun-2007. 